Check for updates

Cite this: Phys. Chem. Chem. Phys., 2021, 23, 7837

Received 21st September 2020 Accepted 10th November 2020

DOI: $10.1039 / \mathrm{d} 0 \mathrm{cp} 04998 \mathrm{k}$

\section{Spatial dimensionality and the binding of small clusters $\uparrow$}

\author{
L. W. Bruch (D)
}

\begin{abstract}
The effect of spatial dimensionality $D$ on the near-threshold binding of small clusters of identical particles is shown. Estimates of the threshold coupling constants for 2 and $N$ bosons are given for $D$ equal to 1 to 5 and the relation to conditions for the Efimov effect is discussed. Variational trial functions for 4 identical spin- $\frac{1}{2}$ fermions in $D=2$ are given.
\end{abstract}

rsc.li/pccp

\section{Introduction}

Helium atoms provide extreme examples of quantum effects in the binding of small atomic clusters. For ${ }^{4} \mathrm{He}$, the dimer, ${ }^{1,2}$ the ground-state trimer, ${ }^{3,4}$ and the Efimov state of the excited trimer ${ }^{5}$ have been detected in experiments with atomic beams. The corresponding clusters ${ }^{3} \mathrm{He}_{2}$ and ${ }^{3} \mathrm{He}_{3}$ of ${ }^{3} \mathrm{He}$ are not selfbound. One direction of research has been to follow the (possible) binding in larger clusters. ${ }^{6-8}$

On the other hand, one may study the relation of the stability of small clusters relative to such self-bound many-body liquids as liquid ${ }^{4} \mathrm{He}$ ( $N$-bosons) and liquid ${ }^{3} \mathrm{He}$ ( $N$-fermions). This turns out to be strongly dependent on the spatial dimension ${ }^{9-11}$ (integer) $D$, as is further discussed here in Section II for $N$-boson thresholds. Related ideas of corresponding states have been used for families of pair potentials in the inert gases, ${ }^{12}$ for the effects of quantum mechanics on the gas-liquid critical point, ${ }^{13}$ and for the evolution of the ground state of a many-body system from gas to liquid to solid as a function of the quantum parameter. ${ }^{9,11}$ The information for the many-fermion system is more limited. There has been a demonstration of dimerization for 4 -fermions near the dimer threshold in two dimensions $(D=2) \cdot{ }^{14}$ In Section III, an extension of the spin algebra for 4 identical spin $-\frac{1}{2}$ fermions ${ }^{15}$ to $D=2$ is given and the relation to estimates based on 2-body thresholds ${ }^{16,17}$ is discussed.

Throughout this paper, atoms of mass $m$ are assumed to interact via central pair potentials. Quantitative results in Section II are given for threshold coupling constants for the Lennard-Jones $(12,6)$ potential,

$$
\phi(r)=4 \varepsilon\left[(\sigma / r)^{12}-(\sigma / r)^{6}\right],
$$

Department of Physics, University of Wisconsin-Madison, Madison,

Wisconsin 53706, USA. E-mail: lwbruch@wisc.edu

$\dagger$ PACS numbers: 36.40.-c, 31.15.ac. with energy minimum $\varepsilon$ and length scale $\sigma$. The coupling constant is defined to be $K=4 m \varepsilon \sigma^{2} / \hbar^{2}$ which is related to the de Boer parameter $\Lambda^{*}$ by $\Lambda^{*}=4 \pi / \sqrt{K}$. This potential is often used as a near-realistic model in corresponding states scalings. ${ }^{11,13}$ Formulation of phase diagrams or critical point parameters as a function of $K$ enables extrapolations and interpolations when estimating the effects of the changes in effective potential that occur in transferring from $D=3$ to an adsorbed monolayer.

Efimov states for three atoms arise for ${ }^{4} \mathrm{He}_{3}$ ref. 5 and in observations of trapped atoms. ${ }^{18}$ There are ${ }^{19,20}$ very weakly selfbound nominally $2 \mathrm{D}$ phases of ${ }^{3} \mathrm{He}$ that have been modeled as many-fermion bound states. ${ }^{21,22}$ While the present paper treats systems of identical particles, it is important to note theoretical treatments of cluster of unequal masses that demonstrate the Efimov effect ${ }^{23}$ may occur in other dimensions. ${ }^{24-27}$

The organization of this paper is as follows: Section II contains the treatment of 2 and $N$ identical spin- 0 bosons and Section III reports results for 4 identical spin $-\frac{1}{2}$ fermions. Section IV contains conclusions.

\section{Two and $N$ bosons}

Efimov $^{23}$ showed that for coupling constants in a very narrow range close to the dimer threshold there is a series of loosely bound excited trimer states. His construction, for three spatial dimensions $(D=3)$, showed that the threshold coupling constant for the ground state trimer is less than that for the dimer. Lim $e t ~ a l .{ }^{28}$ demonstrated the existence of a very weakly bound (ca. $1 \mathrm{mK}$ ) excited state of ${ }^{4} \mathrm{He}_{3}$ and later calculations ${ }^{5}$ confirmed that the excited state does depend on coupling constant as predicted by Efimov. However, although the Efimov effect of three identical bosons does not occur in two spatial dimensions $(D=2)$, there does exist a weakly bound trimer excited state near 
the dimer threshold ${ }^{29,30}$ and universality conditions apply for finite range potentials. ${ }^{30,31}$

It is noteworthy that a variational calculation of the $\mathrm{N}$-boson threshold coupling constant coincides with the dimer threshold in $D=2^{9}$ and $D=1 .^{10}$ Studies in fractional dimension $\tilde{D}$ show $^{27,30}$ that the Efimov effect occurs only for $2.3<\tilde{D}<3.8$. Here, variational estimates ${ }^{32}$ of the 2-boson and $N$-boson threshold will be given for dimension $D=1,2,3,4,5$. The pair potential is the $\mathrm{LJ}(12,6)$ model of eqn (1) and the $N$-boson variational trial function has the Jastrow form

$$
\Psi_{0, J}=\prod_{i<j} f\left(r_{i j}\right)
$$

with factors $f$ of the form

$$
f(r)=\exp \left[-(b / r)^{s}\right]
$$

the length scale $b$ and the power $s$ are variational parameters. A cluster expansion of the trial energy $E_{\mathrm{t}}$ for $N$ bosons in the $D$-dimensional volume $\Omega$ gives a low density estimate:

$$
E_{\mathrm{t}}=\frac{1}{2} \frac{N}{\Omega} \int \mathrm{d}^{D} r\left\{\phi(r) f(r)^{2}+\frac{\hbar^{2}}{m}(\nabla f)^{2}\right\}+\ldots
$$

subject to

$$
\left|\int \mathrm{d}^{D} r\left[f(r)^{2}-1\right]\right|<\infty .
$$

The integral in eqn (4) also appears in the expectation value for the dimer trial energy but using a trial function $\psi(r)$ subject to a different boundary condition than $f(r)$. The form used here is

$$
\psi(r)=f(r) / r^{\eta},
$$

where $\eta$ depends on $D$ and the angular momentum; it is chosen to make the large- $r$ asymptote of $\psi$ match to the subdominant solution to the zero energy two-body Schrödinger equation. Upper bounds on the threshold coupling constants for 2 and $N$ bosons are obtained by locating the coupling constant $K$ that makes the corresponding $r$-integrals equal zero after optimization of $b$ and $s$. With these trial functions, the integrals are done analytically in terms of $\Gamma$-functions. ${ }^{32}$ More generally this condition may also be satisfied by integrating a two-body zero-energy Schrödinger equation, but in the cases where that has been done, the results from the variational calculation are within $0.1 \%$ of those of the numerical integrations. Results are shown for the 2 (S - wave) and $N$-boson thresholds for $D=1$ to 5 in Table 1 . Additionally the thresholds for dimers with 1 and 2 units of angular momentum ("P" and "D") in 2 and 3 dimensions are given in Table 2, where it may be seen that the simple correlation factor eqn (3) gives a fine approximation.

It is remarkable that the 2 and $N$-boson threshold are identical in $D=1$ and 2 and that the difference increases for higher dimension. Although the $N$-boson values are based on the Jastrow trial function, there is no example showing that this fails to locate the threshold in $D=2$. A search for the Efimov effect for 3 identical bosons in $D=2$, which would have demonstrated a failure of the Jastrow form in $D=2$, was unsuccessful $^{29}$ and later work ${ }^{27,30}$ reinforced the conclusion
Table 1 Variational estimates of threshold coupling constants $K_{2}$ and $K_{N}$ for the $2(L=0, S$-wave) and many-boson ground states with the LennardJones $(12,6)$ pair potential in $D$ dimensions. $\eta$ is the power law for the dimer trial function

\begin{tabular}{llll}
\hline$D$ & $\eta$ & $K_{2}$ & $K_{N}$ \\
\hline 1 & 0 & 22.368 & $22.368^{a}$ \\
2 & 0 & $14.753^{b c}$ & $14.753^{c}$ \\
3 & 1 & 22.368 & $8.676^{d}$ \\
4 & 2 & 31.54 & 4.16 \\
5 & 3 & 42.23 & 1.218
\end{tabular}

${ }^{a}$ Krotscheck et al. ${ }^{10 b}$ Using $^{14} \eta=1 / 2$ leads to $K_{2}=17.96$, and does not give a bound ${ }^{3} \mathrm{He}_{2}$, for which $K \simeq 16.6$. ${ }^{c}$ Miller and Nosanow ${ }^{9}$ and $\mathrm{Cabral}^{33}$ give for the Schrödinger solution $K_{2}=K_{N}=14.744 .{ }^{d}$ Schrödinger

\begin{tabular}{|c|c|c|c|c|}
\hline$D$ & $\eta_{\mathrm{P}}$ & $K_{\mathrm{P}}$ & $\eta_{\mathrm{D}}$ & $K_{\mathrm{D}}$ \\
\hline 2 & 1 & $31.54^{a}$ & 2 & 54.35 \\
\hline 3 & 2 & $42.23^{b}$ & 3 & $68.23^{b}$ \\
\hline
\end{tabular}
solutions are $K_{2}=22.362$ and $K_{N}=8.6725$.

Table 2 Dimer threshold coupling constants for the Lennard-Jones(12,6) pair potential for states with 1 ("P") and 2 ("D") units of orbital angular momentum, using the dimer trial functions of eqn (7)

that the effect is absent in $D=2$ and also showed it is absent for $D=4$. Thus the results for $D=4$ and 5 in Table 1 show that another mechanism must be acting to separate the 2 and $N$ boson thresholds there.

\section{Binding of four identical fermions}

Quantum statistics effects, arising from the requirement of antisymmetric total wave functions, lead to more complex analyses for identical spin- $\frac{1}{2}$ fermions. ${ }^{14,15,33,34}$ For two such particles, the spatial component of the ground state wave function can be the same as for bosons of the same mass, with the antisymmetry arising from the spin component. Already for three and four fermions, the mixing of space and spin components introduces enough complexity that there are few systematic studies available for four fermions. ${ }^{14,34}$ Bounds on the ground state energy using 2-body comparison problems, the Hall-Post inequalities, ${ }^{16,17}$ provide some indirect information. Here results for 4 -fermions in $D=3$ are extended to $D=2$ (a Cartesian $x-y$ plane) and a comparison is given to results for Hooke's law oscillators.

Jacobi coordinates for 4 identical particles are

$$
\begin{aligned}
\mathbf{R} & =\left(\mathbf{r}_{1}+\mathbf{r}_{2}+\mathbf{r}_{3}+\mathbf{r}_{4}\right) / 4 \\
\mathbf{u} & =\frac{1}{2}\left(\mathbf{r}_{1}+\mathbf{r}_{2}\right)-\frac{1}{2}\left(\mathbf{r}_{3}+\mathbf{r}_{4}\right) \\
\mathbf{v} & =\mathbf{r}_{1}-\mathbf{r}_{2} \\
\mathbf{w} & =\mathbf{r}_{3}-\mathbf{r}_{4}
\end{aligned}
$$

The states of total spin $S$ for 4 spin- $\frac{1}{2}$ fermions are one of $S=2$ (degeneracy 5), three of $S=1$ (each of degeneracy 3) and two of $S=0$ (each non-degenerate). The total wave function for 
$S=2$ is a product of space and spin factors, but for $S=1$ and $S=0$ the total wave function is a sum of factored pieces. The requirements imposed by antisymmetry of the total wave function have been given elsewhere. ${ }^{15}$ For $D=3$, trial wave functions based on the Jastrow product eqn (2) and factors with $0(S=2), 1(S=1)$ and 2 or $0(S=0)$ units of orbital angular momentum have been given. ${ }^{15}$

Analytical results for the 4-body problem using the Hooke's law pair potential,

$$
\Phi=\sum_{i<j} \frac{1}{2} k\left(\mathbf{r}_{i}-\mathbf{r}_{j}\right)^{2},
$$

are known in terms of normal modes. The internal Jacobi coordinates $u, v, w$ all have oscillator frequencies $\omega_{4}=\sqrt{4 k / m}$, while the frequency for a dimer of reduced mass $\mu$ is $\omega_{2}=\sqrt{k / \mu}$. The (Hall) lower bounds ${ }^{15,16}$ in terms of the ground state and first odd parity excited state energies of two-body problems with effective mass $\mu$, $E_{0}(2, \mu)$ and $E_{1}(2, \mu)$ are

$$
\begin{gathered}
E_{0}(4 f, S=2) \geq 6 E_{1}(2, m)=3 \hbar \omega_{4}[1+(D / 2)] . \\
E_{0}(4 f, S=1) \geq 2\left[E_{0}(2, m)+2 E_{1}(2, m)\right]=\hbar \omega_{4}[2+(3 D / 2)] . \\
E_{0}(4 f, S=0) \geq 3\left[E_{0}(2, m)+E_{1}(2, m)\right]=\hbar \omega_{4}[(3 / 2)(D+1)] .
\end{gathered}
$$

The final equalities in these equations are the values obtained for Hooke's law oscillators in $D$-dimensions. The analytic solution for Hooke's law oscillators achieves the lower bounds in $D=3$ for $S=2$ and $S=1$ but not for $S=0$. For the 3-fermion cases, the corresponding lower bounds are achieved ${ }^{15}$ for both $S=\frac{3}{2}$ and $S=\frac{1}{2}$. The lower bound for 4 bosons, $E_{0}(4 b) \geq 6 E_{0}(2, m)$ is also achieved. ${ }^{16}$

In extending the results ${ }^{15}$ to $D=2$, two difficulties arise: (1) the spatial component of the $S=2$ trial function is a determinant that does not adapt to $D=2$. (2) The spatial factors of nonzero angular momentum in $D=3$ are given in terms of a real component of the spherical harmonics but that does not arise in $D=2$. Resolutions to both difficulties are given here.

To begin a resolution of (1), a totally antisymmetric spatial wave function factor $F_{2}$ for the $S=2$ case is constructed with 4 factors of the internal Jacobi coordinates. It is, for Cartesian components of the Jacobi coordinates $\alpha$ to $\delta$ chosen to be $x$ or $y$ of $D=2$,

$$
\begin{aligned}
F_{2}(\alpha \beta, \gamma \delta)= & u_{\alpha} u_{\beta}\left(v_{\gamma} w_{\delta}+v_{\delta} w_{\gamma}\right) \\
& -\tau_{\alpha} \tau_{\beta}\left(-2 u_{\gamma} u_{\delta}+2 \sigma_{\gamma} \sigma_{\delta}\right) \\
& -\sigma_{\alpha} \sigma_{\beta}\left(2 u_{\gamma} u_{\delta}-2 \tau_{\gamma} \tau_{\delta}\right)
\end{aligned}
$$

with $\sigma_{\alpha}=\left(v_{\alpha}+w_{\alpha}\right) / 2$ and $\tau_{\alpha}=\left(v_{\alpha}-w_{\alpha}\right) / 2$. However this is not an angular momentum eigenstate. The two trial functions

$$
\begin{aligned}
& \Psi(0, F)=\left[F_{2}(x y, x x)-F_{2}(x y, y y)\right] \Psi_{0, J} \\
& \Psi(4, F)=\left[F_{2}(x x, x y)+F_{2}(y y, x y)\right] \Psi_{0 . J}
\end{aligned}
$$

are totally antisymmetric wavefunctions that are eigenfunctions of $L_{z}^{2}$ ( $L_{z}$ is the $z$-component of total angular moment) with eigenvalues $\ell_{z}^{2} \hbar^{2}$, for $\ell_{z}=0$ and 2 , respectively.
The resolution of (2) for $D=2$ needed for $\ell_{z} \neq 0$, which also applies in adapting trial functions presented for $D=3$, is to form the combination

$$
\Psi\left(\ell_{z}\right)=\left[\ell_{z} \hbar+L_{z}\right] \Psi\left(\ell_{z}^{2}, F\right)
$$

which is an antisymmetric trial function with $L_{z}$ eigenvalue $\ell_{z} \hbar$. $\Psi\left(\ell_{z}^{2}, F\right)$ for $D=2$ is given in eqn (14). Although this trial function is formally complex, expectation values reduce to real-number calculations, e.g.,

$$
\left(\Psi\left(\ell_{z}\right), H \Psi\left(\ell_{z}\right)\right)=2 \ell_{z}^{2} \hbar^{2}\left(\Psi\left(\ell_{z}^{2}, F\right), H \Psi\left(\ell_{z}^{2}, F\right)\right)
$$

for a Hamiltonian $H$ that commutes with $L_{z}$. Then the wave functions of eqn (13) and (14) lead to variational estimates $E(4 f, S=2)_{\mathrm{t}}=7 \hbar \omega_{4}$ for the Hooke's law model and do not achieve the lower bound $6 \hbar \omega_{4}$ of eqn (9). To summarize, the lower bound for $S=2$ is achieved for $D \geq 3$ and for $S=1$ is achieved for $D \geq 2$, but the lower bound for $S=0$ is not achieved for either $D=3$ or $D=2$; in fact, $E_{0}(4 f, S=0)=\hbar \omega_{4}[(3 D / 2)+2]$. The general inequalities are satisfied in these cases though.

There is limited computational experience with such fermion trial functions. Lim and co-workers ${ }^{34}$ treated the $S=0$ case of ${ }^{3} \mathrm{He}_{4}$ in $D=3$ and found no self-binding. They did find that the $L=2$ configuration seemed to be closer to binding than the $L=0$ configuration and interpreted this difference as arising from wave function nodes that occur as a function of angle rather than as a function of radius. A similar phenomenon happens ${ }^{35}$ for 3 boson in $D=3$. Vranjes and Kilic ${ }^{14}$ treated the $S=0, L=0$ case in $D=2$ and found dimerization ( $2 S=0$ dimers) for ${ }^{3} \mathrm{He}$ parameters, but not a self-bound configuration of ${ }^{3} \mathrm{He}_{4}$. That result is consistent with the conclusion of Miller and Nosanow ${ }^{9}$ that the threshold for many-fermion self-binding is significantly larger than the dimer threshold.

It is not known what if any connection there is between these results and the dimerization observed for trapped atoms. $^{36}$ The corresponding question in nuclear physics, the stability of the tetra-neutron, remains unsettled. ${ }^{37}$

\section{Conclusions}

The merging of threshold coupling constants for 2 and $N$ bosons in $D=1$ and $D=2$ correlates with the absence of the Efimov effect there. However the Efimov effect is absent also for $D=4$ and $D=5$ while the 2 and $N$ boson threshold coupling constants are well-separated there. Whether this contrast is coincidental is not known. It may be clarified with extensions of the construction originally used by Efimov $^{23}$ to demonstrate the existence of long-range effective interactions.

Hooke's law springs have provided many examples where the Hall-Post lower bounds on the ground state energies of few-body systems are attained. The 4 -fermion system in $D=2$ and $D=3$ provides cases where the lower bound is not attained. More systematic studies using group theoretic constraints for few-fermion systems near threshold appear to be warranted, especially as there is a self-bound state of ${ }^{3} \mathrm{He}$ on graphite that has a very low areal density. ${ }^{19,22}$ 


\section{Conflicts of interest}

There are no conflicts to declare.

\section{References}

1 F. Luo, G. C. McBane, G. Kim, C. F. Giese and W. R. Gentry, J. Chem. Phys., 1993, 98, 3564-3567.

2 W. Schöllkopf and J. P. Toennies, Science, 1994, 266, 1345-1348.

3 L. W. Bruch, W. Schöllkopf and J. P. Toennies, J. Chem. Phys., 2002, 117, 1544-1566.

4 J. Voigtsberger, S. Zeller, J. Becht, N. Neumann, F. Sturm, H.-K. Kim, M. Waitz, F. Trinter, M. Kunitski, A. Kalinin, J. Wu, W. Schöllkopf, D. Bressanini, A. Czasch, J. B. Williams, K. Ullmann-Pfleger, L. Ph. H. Schmidt, M. S. Schöffler, R. E. Grisenti, T. Jahnke and R. Dörner, Nat. Commun., 2014, 5, 5765.

5 M. Kunitski, S. Zeller, J. Voigtsberger, A. Kalinin, L. Ph. H. Schmidt, M. Schöffler, A. Czasch, W. Schöllkopf, R. E. Grisenti, T. Jahnke, D. Blume and R. Dörner, Science, 2015, 348, 551-555.

6 M. Lewerenz, J. Chem. Phys., 1997, 106, 4596-4603; E. Krotscheck and R. Zillich, J. Chem. Phys., 2001, 115, 10161-10174.

7 V. R. Pandharipande, S. C. Pieper and R. B. Wiringa, Phys. Rev. B: Condens. Matter Mater. Phys., 1986, 34, 4571-4582.

8 E. Sola, S. Casulleras and J. Boronat, Phys. Rev. B: Condens. Matter Mater. Phys., 2006, 73, 092515.

9 M. D. Miller and L. H. Nosanow, J. Low Temp. Phys., 1978, 32, 145-157.

10 E. Krotscheck, M. D. Miller and J. Wojdylo, Phys. Rev. B: Condens. Matter Mater. Phys., 1999, 60, 13028-13037.

11 L. H. Nosanow, L. J. Parish and F. J. Pinski, Phys. Rev. B: Solid State, 1975, 11, 191-204.

12 K. T. Tang and J. P. Toennies, J. Chem. Phys., 2003, 118, 4976-4983.

13 J. de Boer and R. B. Bird, in Molecular Theory of Gases and Liquids, ed. J. O. Hirschfelder, C. F. Curtiss and R. B. Bird, Wiley, New York, 1965, ch. 10.

14 L. Vranjeŝ and S. K. Kilić, Phys. Rev. A: At., Mol., Opt. Phys., 2002, 65, 042506.
15 L. W. Bruch, J. Chem. Phys., 1980, 72, 5511-5515.

16 R. L. Hall, J. Phys. A: Gen. Phys., 1968, 1, 468-474.

17 J. M. Richard, A. Volcarce and J. Vijande, Ann. Phys., 2020, 412, 168009.

18 R. Grimm, Few-Body Syst., 2019, 60, 23.

19 D. Sato, K. Naruse, T. Matsui and H. Fukuyama, Phys. Rev. Lett., 2012, 109, 25306.

20 S. Nakamura, K. Matsui, T. Matsui and H. Fukuyama, Phys. Rev. B, 2016, 94, 180501.

21 M. Ruggeri, E. Vitali, D. E. Galli, M. Boninsegni and S. Morono, Phys. Rev. B, 2016, 93, 104102.

22 M. C. Gordillo and J. Boronat, Phys. Rev. Lett., 2016, 116, 145301.

23 V. Efimov, Phys. Lett. B, 1970, 33, 563-564; Sov. J. Nucl. Phys., 1971, 12, 589-595 [Yad. Fiz., 1970, 12, 1080-1091].

24 S. Moroz, J. P. D'Inaco and D. S. Petrov, Phys. Rev. Lett., 2015, 115, 180406.

25 M. T. Yamahsita, Few-Body Syst., 2019, 60, UNSP22.

26 Y. Nishida, Phys. Rev. Lett., 2017, 118, 230601.

27 D. E. Rosa, T. Frederico, G. Kreim and M. T. Yamashita, Phys. Rev. A, 2018, 97, 050701.

28 T. K. Lim, K. Duffy and W. C. Damert, Phys. Rev. Lett., 1977, 38, 341-343.

29 L. W. Bruch and J. A. Tjon, Phys. Rev. A: At., Mol., Opt. Phys., 1979, 19, 425-432.

30 E. Nielsen, D. V. Fedorov, A. S. Jensen and A. Garrido, Phys. Rep., 2001, 347, 373-459.

31 D. Blume, Phys. Rev. B: Condens. Matter Mater. Phys., 2005, 72, 095410.

32 L. W. Bruch, Phys. Rev. B: Solid State, 1976, 13, 2873-2876; 1984, 30, 6769(E).

33 F. Cabral and L. W. Bruch, J. Chem. Phys., 1979, 70, 4669-4672.

34 S. Nakaishi-Maeda, T. K. Lim and Y. Akaishi, J. Chem. Phys., 1982, 77, 5581-5583.

35 L. W. Bruch, J. Chem. Phys., 1999, 110, 2410-2413.

36 M. Yu. Kagan and A. V. Turlapov, Phys.-Usp., 2019, 62, 215-248.

37 M. D. Higgins, C. H. Greene, A. Kievsky and M. Viviani, Phys. Rev. Lett., 2020, 125, 052501; O. Ivanytskyi, M. Angeles Perez Garcia and C. Albertus, Eur. Phys. J. A, 2019, 55, 184; M. Kisamori, et al., Phys. Rev. Lett., 2016, 116, 052501. 